\title{
Experimental Study of the Mechanical Properties of a Novel Supramolecular Polymer Filament Using a Microtensile Tester Based on Electronic Balance
}

\author{
Y. Huan ${ }^{1}$, Y.Q. Shao ${ }^{1}$, Y.J. Dai ${ }^{1}$, Y.Q. Liü ${ }^{2}$, T.Y. Wang ${ }^{2}$, T.H. Zhang ${ }^{3}$, and M.H. Liu ${ }^{2}$ \\ 1 State Key Laboratory of Nonlinear Mechanics (LNM), Institute of Mechanics, Chinese Academy of Sciences, Beijing, China \\ 2 Beijing National Laboratory for Molecular Science (BNLMS), CAS Key Laboratory of Colloid, Interface and Chemical Thermodynamics, Institute of \\ Chemistry, Chinese Academy of Sciences, Beijing, China \\ 3 College of Mechanical Engineering, Zhejiang University of Technology, Hangzhou, China
}

Keywords

Supramolecular polymer, Free standing, Nanotubes, Mechanical properties, Tensile test

\section{Correspondence}

Huan Yong,

State Key Laboratory of Nonlinear Mechanics (LNM),

Institute of Mechanics,

Chinese Academy of Sciences,

No. 15 Beisihuanxi Road,

Beijing 100190,

China

Email: huany@Inm.imech.ac.cn

Received: April 3, 2014;

accepted: June 18, 2014

doi:10.1111/ext.12114

\begin{abstract}
A novel kind of supramolecular polymer filament with a diameter of $2-5 \mu \mathrm{m}$ was artificially synthesized, which is in fact a bundle of self-assembled nanotubes with a diameter of about $40 \mathrm{~nm}$. The filament can be drawn from a special alkaline aqueous solution directly and free standing in room temperature, which has never been performed earlier. A microtensile tester was developed with the aid of an electronic balance to investigate the mechanical properties of the new filament. Monotonic and load-unload tensile tests were performed, respectively. The maximum tensile strength and the elastic modulus of the filament were $23.8 \mathrm{MPa}$ and $1.9 \mathrm{GPa}$, respectively, which were higher than previous supramolecular polymers and comparable to some covalent-linked polymers.
\end{abstract}

\section{Introduction}

Supramolecular polymers are made by the selfassembly of many monomeric units with directional and reversible non-covalent bonds. ${ }^{1}$ They have a wide range of applications in biomaterials, ${ }^{2-4}$ smart materials, ${ }^{5,6}$ medicines, ${ }^{7-9}$ electronic devices, ${ }^{10,11}$ and optical technologies. ${ }^{12,13}$ Because of their highly organized nanostructures, supramolecular polymers present many advantages, such as molecular operability, cycle applicability, self-healing properties, and molecular recognition. ${ }^{14}$ However, until now, most supramolecular polymer filaments are not free standing, which may bring out poor mechanical properties and may limit their applications.

In this paper, a kind of free-standing supramolecular polymer filament, with a diameter of $2-5 \mu \mathrm{m}$, was artificially synthesized. We have found that an L-histidine-terminated bolaamphiphile could self-assemble into supramolecular polymer filaments in slightly alkaline aqueous solution (pH 8-9). Then, the supramolecular polymer filament could be drawn from the aqueous solution by a needle directly. It is in fact a bundle of hollow nanotubes self-assembled by non-covalent bonding, which may mean a weaker tensile strength. However, the phenomenon of free standing indicates a better tensile strength. Further experimental study is needed to investigate the novel supramolecular polymer filament.

However, the test force should be very tiny (much less than $10^{-3} \mathrm{~N}$ ), estimated according to the diameter of the filament and the strength of some superpolymers. The commercial material testing machines, such as INSTRON 5848 Micro Tester (INSTRON, Norwood, MA, USA) ${ }^{15}$ and SIROLANTESTER (CSIRO, Australia), ${ }^{16}$ have no sufficient force resolution for this test due to the strain-gaged force transducer. 
In order to acquire enough force resolution, more sensitive elastic components are adopted as a substitute for the strain-gaged force transducer, such as the atomic force microscope (AFM) cantilever, ${ }^{17-20}$ microelectromechanical system (MEMS)-based cantilever $^{21-23}$ and other specially designed film cantilevers. $^{24,25}$ These cantilevers have good force resolution and are suitable for the tensile tests of microscale filaments. However, it is difficult to install the specimen because the sensitive elastic component is fragile and small in size.

In addition to the above apparatuses, another kind of apparatus is designed based on electromagnetism. A coil-magnet component is adopted as actuator, as well as force transducer. ${ }^{26-30}$ In this way, the tiny force can be easily measured by recording the currents in the coil. In fact, there are two kinds of design, namely moving coil or moving magnet. The former have a better force linearity in a long displacement range than the latter. ${ }^{26}$ This technique has been applied commercially, such as MTS NanoBionix ${ }^{31,32}$ (now taken over by Agilent, Santa Clara, CA, USA). It has the distinct advantage of perfect force resolution, low hysteresis, and easy control. However, a spring with a stiffness of about $80 \mathrm{~N} / \mathrm{m}$ is used to support the moving mass in order to avoid bearing friction, which seriously limits the testing ability of small stiffness specimens. ${ }^{33}$ In this paper, a new microtensile tester was developed with the aid of an electronic balance in order to test the novel supramolecular polymer filament. This tester is suitable for tensile test of microspecimens with very low stiffness, and is easier to manipulate. Moreover, the electronic balance has ideal force accuracy without additional calibration.

\section{Experiments}

\section{Specimen}

In slightly alkaline aqueous solution ( $\mathrm{pH} 8-9), \mathrm{EDH}$ ( $N, N$-eicosanedioyl-di-L-histidine) can assemble into extremely long supramolecular nanotubes with a length-to-diameter ratio of more than 5000. These nanotubes are randomly distributed and nicely separated from each other. The internal diameters and wall thicknesses of nanotubes are about 22 and $10-14 \mathrm{~nm}$, respectively. When a needle is put into the solution and carefully lifted out of the liquid surface, a supramolecular filament with length of about several centimeters can be dragged out directly. More details are described in Ref. 34. The SEM inspections show that the filament has an approximately uniform diameter of $2-5 \mu \mathrm{m}$ (Fig. 1(a)). The magnified view demonstrates that the filament is constructed by many well-aligned hollow nanotubes. The microstructure is illustrated in Fig. l(b). Each supramolecular polymer filament is speculated to contain at least 2000 nanotubes as the single nanotube's outer diameter is about $40 \mathrm{~nm}$.

\section{Apparatus}

In order to investigate the mechanical properties of the novel supramolecular polymer filaments, a microtensile tester was constructed using an electronic balance (Fig. 2).

The actuator of the tester is a step motor, which has a step of about $1.2 \mu \mathrm{m}$ per pulse with a stroke of $30 \mathrm{~mm}$. The force is measured by using an electronic balance with a readability of $0.1 \mathrm{mg}$ and a measurement range of $120 \mathrm{~g}$. This means that the force resolution is about $1 \mu \mathrm{N}$ and the force range is about 1.2 N. More details of force measurement will be described in the following. The displacement of the step motor is measured by a linear variable differential transformer (LVDT) with a resolution of $0.1 \mu \mathrm{m}$ in $2 \mathrm{~mm}$ displacement range. It should be noted that the electronic balance must be isolated from the tester's main frame in order to avoid the motor's vibration.

The upper grip is a metal hook linked to the core rod of LVDT, whereas the lower grip is settled on the electronic balance. Before testing, the filament is attached to the upper and lower grips with a relaxed state. At this time, the reading of the balance is the mass $M$ of lower grip. Then, the step motor drives the upper grip, the filament specimen is stretched, and the reading of the balance will be changed to $M_{\mathrm{N}}$. The force $F$ applied on the filament can be easily calculated by Eq. (1):

$$
F=\left(M-M_{N}\right) g
$$

where the gravity acceleration $g$ at Beijing, China, $39^{\circ} 55^{\prime} \mathrm{N}$ and $116^{\circ} 55^{\prime} \mathrm{E}$, is $9.8012 \mathrm{~N} / \mathrm{kg}$.

At the same time, the displacement of the upper grip is measured by the LVDT, which is regarded as the extension of filament.

\section{Testing procedure}

Firstly, the supramolecular polymer filament was drawn from the aqueous solution by using a needle, and then rolled to the grips directly. It should be noted that the filament specimen must be kept relaxed before extension. The gage length of the specimen was about $10 \mathrm{~mm}$. Special care must be taken to align the filament accurately between the two grips. 
(a)

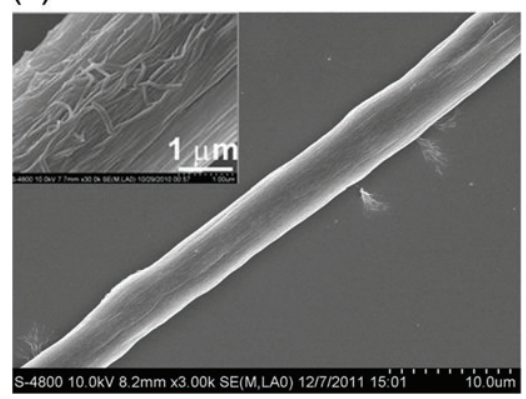

(b)

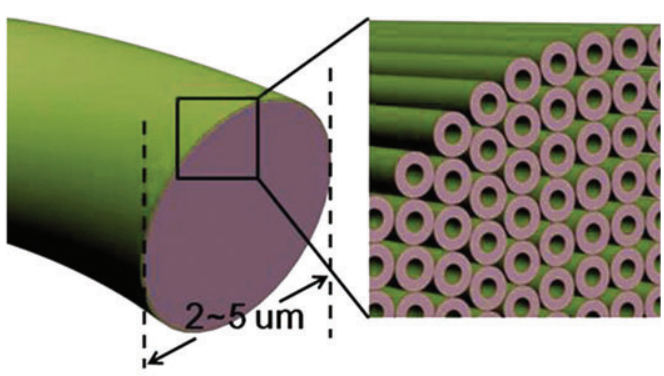

Figure 1 Illustrations of the supramolecular polymer filament. (a) SEM photograph and (b) structure diagram in microscale. The filament is actually constructed by many well-aligned hollow nanotubes.

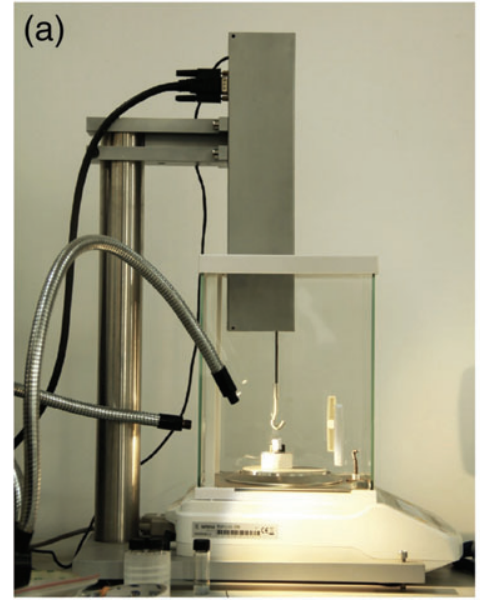

(b)

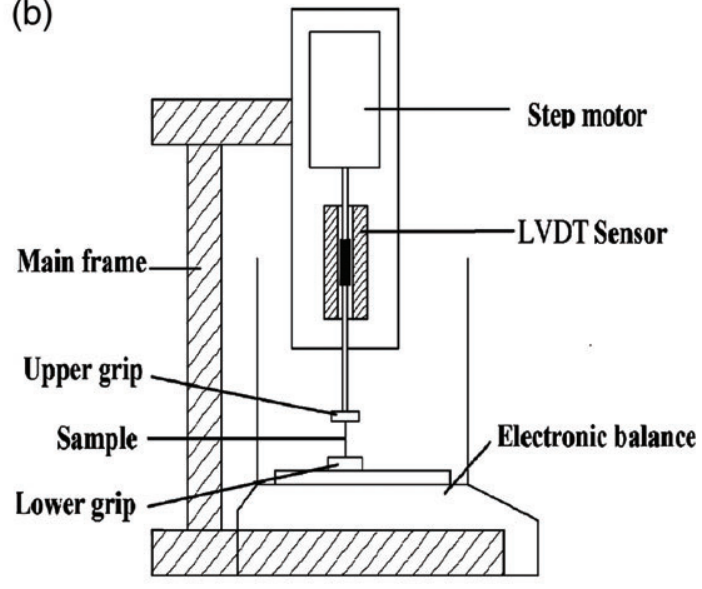

Figure 2 Illustration of the experimental apparatus. (a) Photograph and (b) schematic arrangement. The force is measured by the electronic balance, and the displacement is measured by the LVDT sensor.
Monotonic tensile tests were carried out on the tester. The force-displacement curves were recorded automatically, and then converted to engineering stress-strain curves by taking the cross sectional area and the gage length into consideration. All the specimens were tested at room temperature and a strain rate of $10^{-3} / \mathrm{s}$. In order to ensure enough balance time, there is 3-s interval between the two steps of motor.

In addition, load-unload tensile tests were carried out at a strain rate of $10^{-3} / \mathrm{s}$ to further investigate the deformation mechanism of the filament. The maximum extension of each load-unload loop was set to increase about $10 \mu \mathrm{m}$ per loop until the filament broke. Similarly the force-displacement curves were recorded automatically, and the corresponding engineering stress-strain curves were obtained.

\section{Results and Discussion}

Monotonic tensile tests

The test results of four specimens are shown in Fig. 3. The results exhibited good linear extension behavior. No plasticity was observed. The slopes of these curves were similar, which indicated the good repeatability of the tests. The mean Young's modulus of about 1.9 GPa was calculated. The maximum tensile strength scattered from 10.9 to $23.8 \mathrm{MPa}$, which was higher than that of previous supramolecular polymers, such as $\mathrm{PCL}_{2000} \mathrm{UPy}_{2} \quad(7 \mathrm{MPa}),{ }^{35}$ and even closed to some covalent-linked superpolymers, such as polypropylene random copolymer (about $30 \mathrm{MPa}) .{ }^{36}$ The scatter of the data may be caused by the uncertain defects of the specimen or the damage of grips.

The failure mode of the supramolecular polymer filaments was brittle fracture, different from most superpolymer filaments. The SEM fractography (Fig. 4) clearly showed that almost all nanotubes were fractured at the same cross section, without any necking and longitudinal splitting. This phenomenon is directly related to the self-assembly microstructure of the non-covalent bond.

Load-unload tensile tests

A typical load-unload curve is shown in Fig. 5. The results clearly showed that the load and unload curve coincided very well, which means a good resilience properties of the filaments. Moreover, it can be deduced that the elongation of the filament results 


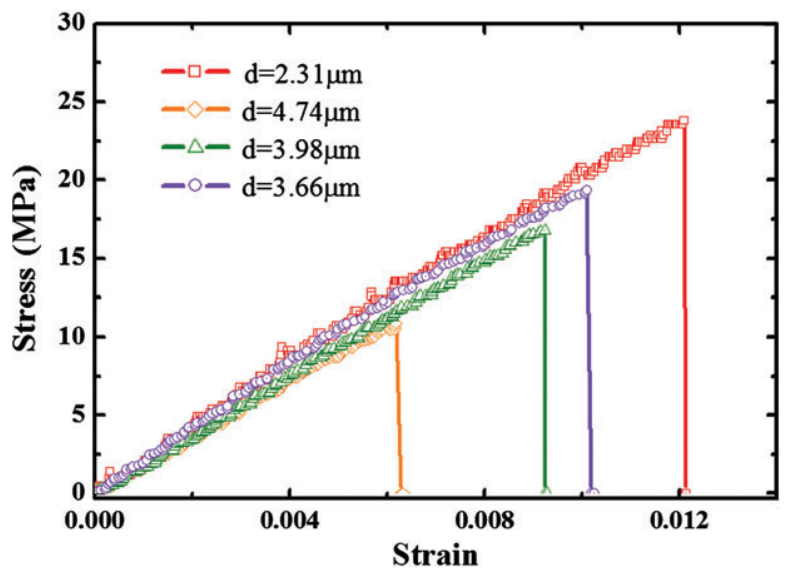

Figure 3 Stress-strain curves of four specimens with different diameters and gage lengths.

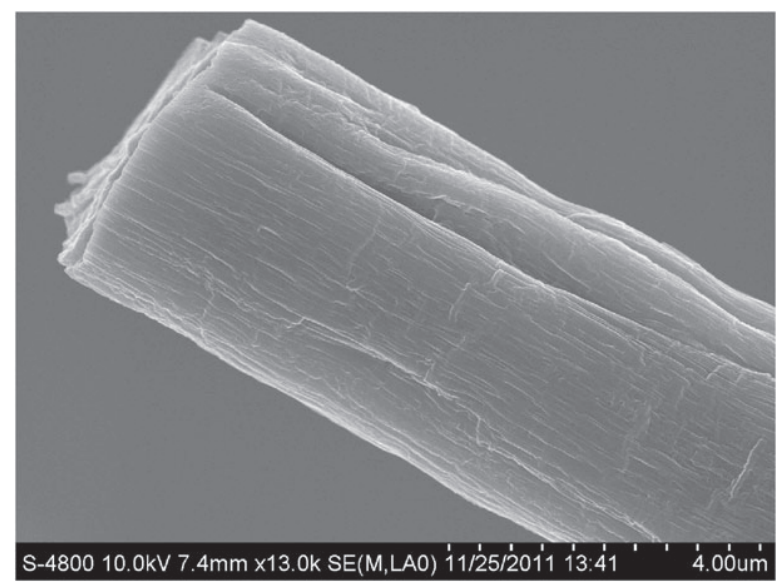

Figure 4 SEM photograph of the fractured specimen.

from the elastic deformation of the nanotubes, rather than sliding among them.

\section{Conclusions}

In this study, a novel kind of free-standing supramolecular polymer filament, with a diameter of $2-5 \mu \mathrm{m}$, was artificially synthesized. A new microtensile tester was developed with the aid of an electronic balance to investigate the mechanical properties of the filament. Monotonic tensile tests and load-unload tensile tests were performed, respectively. The specimens exhibited good linear extension behavior and good resilience without any plasticity. The failure mode was brittle fracture. Of note was the maximum tensile strength and the elastic modulus of the filament were as high as $23.8 \mathrm{MPa}$ and $1.9 \mathrm{GPa}$,

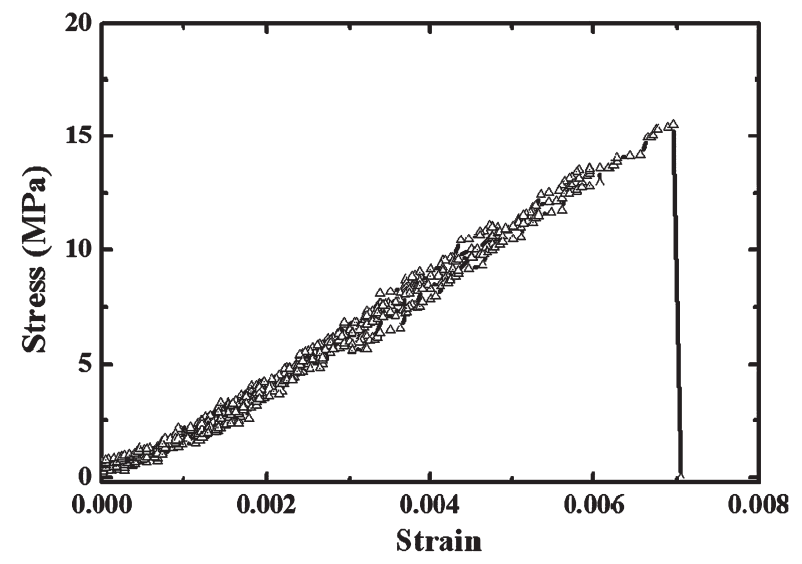

Figure 5 Load-unload tensile test results of the supramolecular polymer filament.

respectively, which were much higher than previous supramolecular polymers and even comparable to some covalent-linked polymers.

\section{Acknowledgments}

The authors gratefully acknowledge Professor He Guowei for his support. This work was supported by the National Natural Science Foundation of China (Grant nos 11372323, 11025212 and 11272318), the project of function development on scientific instruments of Chinese Academy of Sciences and Opening Fund of State Key Laboratory of Nonlinear Mechanics.

\section{References}

1. Brunsveld, L., Folmer, B.J.B., Meijer, E.W., and Sijbesma, R.P., "Supramolecular polymers," Chemical Reviews 101: 4071-4098 (2001).

2. Yang, Z., Liang, G., Ma, M.L., Gao, Y., and Xu, B., "In vitro and in vivo enzymatic formation of supramolecular hydrogels based on self-assembled nanofibers of a $\beta$-amino acid derivative," Small 3: 558-562 (2007).

3. Wang, Y.X., and Shen, J.C., "Progress in non-viral gene delivery systems fabricated via supramolecular assembly," Chinese Science Bulletin 50(4): 289-294 (2005).

4. Hartgerink, J.D., Beniash, E., and Stupp, S.I., "Self-assembly and mineralization of peptideamphiphile nanofibers," Science 294: 1684-1688 (2001).

5. Zubarev, E.R., Pralle, M.U., Sone, E.D., and Stupp, S.I., "Scaffolding of polymers by supramolecular nanoribbon," Advanced Materials 14: 198-203 (2002). 
6. Kishimura, A., Yamashita, T., Yamaguchi, K., and Aida, T., "Rewritable phosphorescent paper by thecontrol of competing kinetic and thermodynamic self-assembling events," Nature Materials 4: 546-549 (2005).

7. Wang, H., Fang, F., Li, X., Fu, C., and Yang, Y.J., "Improved photostability of Vitamin A palmitate originating from self-assembled supramolecular gels," Chinese Science Bulletin 57(33): 4257-4263 (2012).

8. Narmoneva, D.A., Vukmirovic, R., Davis, M.E., Kamm, R.D., and Lee, R.T., “Endothelial cells promote cardiac myocyte survival and spatial reorganization implications for cardiac regeneration," Circulation 110: 962-968 (2004).

9. Rajangam, K., Behanna, H.A., Hui, M.J., Han, X.Q., et al., "Heparin binding nanostructures to promote growth of blood vessels," Nano Letters 6: 2086-2090 (2006).

10. Ajayaghosh, A., Varghese, R., George, S.J., and Vijayakumar, C., "Transcription and amplification of molecular chirality to oppositely biased supramolecular $\pi$ helices," Angewandte Chemie 45: 1141-1144 (2006).

11. Mao, C.B., Solis, D.J., Reiss, B.D., et al., "Virus-based toolkit for the directed synthesis of magnetic and semiconducting nanowires," Science 303: 213-217 (2004).

12. Yagai, S., Iwashima, T., Kishikawa, K., Nakahara, S., Karatsu, T., and Kitamura, A., "Photoresponsive self-assembly and self-organization of hydrogen-bonded supramolecular tapes," Chemistry - A European Journal 12: 3984-3994 (2006).

13. Eastoe, J., Sanchez-Dominguez, M., Wyatt, P., and Heenan, R. K., "A photo-responsive organogel," Chemical Communications 22: 2608-2609 (2004).

14. Aida, T., Meijer, E.W., and Stupp, S.I., "Functional supramolecular polymers," Science 33: 5813-5817 (2012).

15. Yang, Y.L., Ma, C.R., Huang, S.J., Jing, L.Q., and Pang, H.T., "Effects of mechanical properties of Kevlar/PTFE fabric-reinforced self-lubricating liners on performance of self-lubricating spherical plain bearings," Applied Mechanics and Materials 29: 197-202 (2010).

16. Yu, W.D., Yan, H.J., and Postle, R., "Estimating blend ratios from the tensile behavior of wool/polyester fiber bundles," Textile Research Journal 74: 198-204 (2004).

17. Yu, M.F., Dyer, M.J., and Skidmore, G.D., "Three-dimensional manipulation of carbon nanotubes under a scanning electron microscope," Nanotechnology 10: 244-252 (1999).
18. Yu, M.F., Lourie, O., Dyer, M.J., Moloni, K., Kelly, T.F., and Ruoff, R.S., "Strength and breaking mechanism of multiwalled carbon nanotubes under tensile load," Science 287: 637-640 (2000).

19. Tan, E.P.S., Goh, C.N., Sow, C.H., and Lim, C.T., "Tensile test of a single nanofiber using an atomic force microscope tip," Applied Physics Letters 86: 1-3 (2005).

20. Li, X.D., Wang, X.N., Chang, W.C., Chao, Y.J., and Chang, M., "Effect of tensile offset angles on micro/nanoscale tensile testing," Review of Scientific Instruments 76: 1-4 (2005).

21. Naraghi, M., Chasiotis, I., Kahn, H., Wen, Y., and Dzenis, Y., "Novel method for mechanical characterization of polymeric nanofibers," Review of Scientific Instruments 78: 1-7 (2007).

22. Demczyk, B.G., Wang, Y.M., Cumings, J., et al., "Direct mechanical measurement of the tensile strength and elastic modulus of multiwalled carbon nanotubes," Materials Science and Engineering A 334: 173-178 (2002).

23. Kiuchi, M., Matsui, S., and Isono, Y., "Mechanical characteristics of FIB deposited carbon nanowires using an electrostatic actuated nano tensile testing device," Journal of Microelectromechanical Systems 16: 191-201 (2007).

24. Eppell, S.J., Smith, B.N., Kahn, H., and Ballarini, R., "Nano measurements with micro-devices: mechanical properties of hydrated collagen fibrils," Journal of the Royal Society Interface 3: 117-121 (2006).

25. Li, X., Su, D., and Zhang, Z., “A novel technique of microforce sensing and loading," Sensors and Actuators A 153: 13-23 (2009).

26. Huan, Y., Zhang, T.H., and Yang, Y.M., "A moving-coil designed micro-mechanics tester with application on MEMS," Measurement Science and Technology 18: 3612-3616 (2007).

27. Hua, T., Xie, H.M., Pan, B., Qing, X.L., Dai, F.L., and Feng, X.Q., “A new micro-tensile system for measuring the mechanical properties of low-dimensional materials-fibers and films," Polymer Testing 26: 513-518 (2007).

28. Skove, M.J., Tritt, T.M., Ehrlich, A.C., and Davis, H.S., "Device for simultaneously measuring stress, strain, and resistance in whiskerlike materials in the temperature range $1.5 \mathrm{~K}<\mathrm{T}<360 \mathrm{~K}$," Review of Scientific Instruments 62: 1010-1014 (1991).

29. Pan, Z.W., Xie, S.S., Lu, L., et al., “Tensile tests of ropes of very long aligned multiwall carbon nanotubes," Applied Physics Letters 74: 3152-3154 (1999).

30. Ding, J.N., Meng, Y.G., and Wen, S.Z., "Scale dependence of tensile strength of micromachined polysilicon MEMS structures due to microstructural 
and dimensional constraints," Chinese Science Bulletin 46(16): 1392-1397 (2001).

31. Tan, E.P.S., Ng, S.Y., and Lim, C.T., "Tensile testing of a single ultrafine polymeric fiber," Biomaterials 26: 1453-1456 (2005).

32. Inai, R., Kotaki, M., and Ramakrishna, S., "Structure and properties of electrospun PLLA single nanofibres," Nanotechnology 16: 208-213 (2005).

33. Huan, Y., Liu, D.X., Yang, R., and Zhang, T.H., "Analysis of the practical force accuracy of electromagnetbased nanoindenters," Measurement 43: 1090-1093 (2010).
34. Liu, Y.Q., Wang, T.Y., Huan, Y., Li, Z.B., He, G.W., and Liu, M.H., "Self-assembled supramolecular nanotube yarn," Advanced Materials 25(41): 5875-5879 (2013).

35. Patricia, Y.W., Dankers, A., Ellen, N.M., et al., "Chemical and biological properties of supramolecular polymer systems based on oligocaprolactones," Biomaterials 27: 5490-5501 (2006).

36. Sahin, S., and Yayla, P., "Effects of testing parameters on the mechanical properties of polypropylene random copolymer," Polymer Testing 24: 613-619 (2005). 